\title{
Complements of Coxeter group quotients
}

\author{
Paolo Sentinelli
}

Received: 13 February 2014 / Accepted: 12 August 2014 / Published online: 26 August 2014

(C) Springer Science+Business Media New York 2014

\begin{abstract}
We consider the complement $W \backslash W^{J}$ of any quotient $W^{J}$ of a Coxeter system $(W, S)$ and we investigate its algebraic, combinatorial and geometric properties, emphasizing its connection with parabolic Kazhdan-Lusztig theory. In particular, we define two families of polynomials which are the analogues, for the poset $W \backslash W^{J}$, of the parabolic Kazhdan-Lusztig and $R$-polynomials. These polynomials, indexed by elements of $W \backslash W^{J}$, have interesting connections with the ordinary Kazhdan-Lusztig and $R$-polynomials.
\end{abstract}

Keywords Coxeter groups $\cdot$ Hecke algebras $\cdot$ P-kernels

\section{Introduction}

Although the poset $W^{J}:=\{w \in W \mid w<s w \forall s \in J \subseteq S\}$ has been well studied and understood both combinatorially and topologically under the Bruhat order of the Coxeter system $\left(W, S\right.$ ) (see [1] and [3]), the complement $W \backslash W^{J}$ does not seem to have been considered yet. In this work we study algebraic, combinatorial and geometric properties of these complements; more precisely, we show that $W \backslash W^{J}$, as a poset under the induced Bruhat order, is graded (Theorem 2.2); the order complex of its intervals is shellable (Theorem 5.4) and we compute explicitly its Möbius function (Theorem 5.6). We show that the study of the poset $W \backslash W^{J}$ arises quite naturally from a construction done by Deodhar in [6] to introduce parabolic Kazhdan-Lusztig theory. In fact Deodhar defined, for an arbitrary Coxeter system $(W, S)$, two $\mathscr{H}$ modules $M^{J, x}$ with a $\mathbb{Z}\left[q^{1 / 2}, q^{-1 / 2}\right]$-basis $\mathscr{B}$ indexed by $W^{J}$, for every $J \subseteq S$, one

P. Sentinelli $(\varangle)$

Dipartimento di Matematica, Università degli Studi di Roma "Tor Vergata", Via della Ricerca

Scientifica, 00133 Roma, Italy

e-mail: paolosentinelli@libero.it 
for each value of a parameter $x \in\{-1, q\}$; here $\mathscr{H}$ is the Hecke algebra of $W$. Algebraically the $\mathscr{H}$-module $M^{J, x}$ can be described as the quotient of the Hecke algebra $\mathscr{H}$ by the annihilator of the basis element $m_{e}^{J, x} \in \mathscr{B}$. We show that this annihilator is an $\mathscr{H}$-module which has a $\mathbb{Z}\left[q^{1 / 2}, q^{-1 / 2}\right]$-basis indexed by $W \backslash W^{J}$ (Corollary 3.3) and is invariant under a certain involution defined in [6]. These facts lead to the construction of two families of elements of $\mathbb{Z}\left[q^{1 / 2}, q^{-1 / 2}\right]$, corresponding to the two $\mathscr{H}$-module structures. These polynomials have an interesting relation with the ordinary $R$-polynomials (Proposition 4.2 ), and we obtain a recursion for their computation (Theorem 4.14). These properties allow us to show the existence of $P$-kernels (as developed by Stanley in [13]) for the poset $W \backslash W^{J}$ (Theorem 7.1). Moreover, this investigation of the annihilator of $m_{e}^{J, x}$ leads to an extension of the parabolic Kazhdan-Lusztig and $R$-polynomials to elements not in the quotient $W^{J}$.

The organization of the paper is as follows. In the next section we fix notation, and we recall some definitions and results about Coxeter groups, their Hecke algebras, parabolic Kazhdan-Lusztig theory, and $P$-kernels. Section 3 is devoted to the study of the annihilator of $m_{e}^{J, x}$ for both the $\mathscr{H}$-modules $M^{J, x}$, and a $\mathbb{Z}\left[q^{1 / 2}, q^{-1 / 2}\right]$-basis is obtained for this $\mathscr{H}$-module. In Sect. 4 we introduce two families of polynomials, as coordinates of an involution on the annihilator of $m_{e}^{J, x}$ with respect to the basis discussed in the previous section. These polynomials are related to the ordinary $R$-polynomials (see Proposition 4.2 ), and a recursion for them is obtained in Theorem 4.14. In Sect. 5 we study the poset $W \backslash W^{J}$ under the induced Bruhat order. We prove that it is a graded poset and that the order complex of its intervals is shellable, and we compute its Möbius function. In Sect. 6 we extend the parabolic KazhdanLusztig and $R$-polynomials, introduced by Deodhar in [6], to elements not in $W^{J}$. Finally, Sect. 7 is devoted to the proof of the existence of $P$-kernels for the poset $W \backslash W^{J}$ and to the existence of the analogues of the Kazhdan-Lusztig polynomials for this poset.

\section{Notations and preliminaries}

In this section we establish some notation, and we collect some basic results in the theory of Coxeter groups and their Hecke algebras which will be useful in the sequel. The reader can consult [2] and [8] for further details.

We let $\mathbb{Z}$ be the ring of integers and $\mathbb{R}$ the field of real numbers. $\mathbb{N}$ is the set of non-negative integers and, if $n \in \mathbb{N},[n]:=\{1,2, \ldots, n\}$; in particular $[0]=\varnothing .|X|$ is the cardinality of a set $X$, and $\subset$ is the proper inclusion between two sets.

Let $(W, S)$ be a Coxeter system. If $v, w \in W$ we define $\ell(v, w):=\ell(w)-\ell(v)$, where $\ell(z)$ is the length of the element $z \in W$. If $J \subseteq S$, we let

$$
\begin{aligned}
W^{J} & :=\{w \in W \mid \ell(s w)>\ell(w) \forall s \in J\}, \\
D_{L}(w) & :=\{s \in S \mid \ell(s w)<\ell(w)\}, \\
D_{R}(w) & :=\{s \in S \mid \ell(w s)<\ell(w)\} .
\end{aligned}
$$


The parabolic subgroup $W_{J} \subseteq W$ is the group with $J \subseteq S$ as generator set. In particular $W_{S}=W$ and $W_{\varnothing}=\{e\}$.

We are interested in $W$ as a poset under the Bruhat order $\leqslant$ (see, e.g. Chapter 2 in [2] or Chapter 5 in [8]). The quotient $W^{J}$ is a subposet of $W$, considered with the induced order. For $v, w \in W^{J}$ such that $v \leqslant w$, the interval $[v, w]^{J} \subseteq W^{J}$ is the set defined by

$$
[v, w]^{J}:=\left\{z \in W^{J} \mid v \leqslant z \leqslant w\right\}
$$

In finite groups $W$ there exists a unique maximal element $w_{0}$, maximal relative to the Bruhat order, and it is the element of maximum length in $W$ (see Section 2.5 in [2]). The following property, known as the lifting property, characterizes the Bruhat order (see Proposition 2.2.7 and Exercise 2.14 in [2]):

Proposition 2.1 Let $v, w \in W$ be such that $v<w$ and $s \in D_{R}(w) \backslash D_{R}(v)$. Then $v \leqslant w s$, and $v s \leqslant w$.

As is well known, the poset $W^{J}$ is graded by the length function (see Theorem 2.5.5 in [2]).

Theorem 2.2 If $u<w$ in $W^{J}$, then there exist elements $w_{i} \in W^{J}$, such that $\ell\left(w_{i}\right)=$ $\ell(u)+i$, for $0 \leqslant i \leqslant k$, and $u=w_{0}<w_{1}<\ldots<w_{k}=w$.

The Möbius function of the poset $W^{J}$ is also known (see Corollary 2.7.10 in [2]).

Proposition 2.3 Let $u, w \in W^{J}$. The Möbius function of the poset $W^{J}$ is

$$
\mu^{J}(u, w)= \begin{cases}(-1)^{\ell(u, w)}, & \text { if }[u, w]^{J}=[u, w], \\ 0, & \text { otherwise. }\end{cases}
$$

For $J \subseteq S$, an element $w \in W$ has a unique expression $w=w_{J} w^{J}$, where $w^{J} \in W^{J}$ and $w_{J} \in W_{J}$ (Proposition 2.4.4 in [2]). The canonical projection $P^{J}$ : $W \rightarrow W^{J}$, defined by

$$
P^{J}(w)=w^{J},
$$

is a morphism of posets (Proposition 2.5.1 in [2]):

Proposition 2.4 Let $v, w \in W$ be such that $v \leqslant w$; then $v^{J} \leqslant w^{J}$.

The following is an immediate consequence of Proposition 2.4 (see also Corollary 2.5.2 in [2]).

Corollary 2.5 Suppose $v \in W^{J}, w \in W, v<w$ and $\ell(v, w)=1$. Then, either $w=s v$, for some $s \in J$, or $w \in W^{J}$.

The next lemma summarizes some known results (see Lemma 3.1 in [5] and Corollary 2.5):

Lemma 2.6 Let $s \in S$ and $w \in W^{J}$. Exactly one of the following three possibilities occurs: 
1. $s \in D_{R}(w)$. In this case $w s \in W^{J}$,

2. $s \notin D_{R}(w)$ and $w s \in W^{J}$,

3. $s \notin D_{R}(w)$ and $w s \notin W^{J}$; in this case $w s=s^{\prime} w$ for a unique $s^{\prime} \in J$.

In the present work we consider the set $W \backslash W^{J}$ with the induced Bruhat order. With $[v, w]^{\backslash J}$ we denote an interval in $W \backslash W^{J}$, i.e. if $v, w \in W \backslash W^{J}$ and $v \leqslant w$,

$$
[v, w]^{\backslash J}:=\left\{z \in W \backslash W^{J} \mid v \leqslant z \leqslant w\right\}
$$

For the topological questions discussed in Sect. 5 it is useful to recall some notation and results about the interval structure of $W$. We refer to Section 2.7 of [2] for this part. Let $\mathscr{M}(u, v)$ be the set of maximal chains in the Bruhat interval $[u, v]$, and let $v=s_{1} s_{2} \ldots s_{q}$ be a reduced expression. We associate with each $\mathbf{m} \in \mathscr{M}(u, v)$ a string of integers $\lambda(\mathbf{m}):=\left(\lambda_{1}(\mathbf{m}), \lambda_{2}(\mathbf{m}), \ldots, \lambda_{k}(\mathbf{m})\right)$, where $k=\ell(u, v)$. Suppose that $\mathbf{m}$ is the chain $v=x_{0} \triangleright x_{1} \triangleright \ldots \triangleright x_{k}=u$. By the Strong Exchange Property (see Theorem 1.4.3 in [2]), $x_{1}=s_{1} s_{2} \ldots \hat{s}_{i} \ldots s_{q}$, where the deleted generator $s_{i}$ is uniquely determined. Let $\lambda_{1}(\mathbf{m}):=i$ and so on. Let $\mathscr{M}^{J}(u, v)$ denote the set of maximal chains in the Bruhat interval $[u, v]^{J}$. Since $\mathscr{M}^{J}(u, v) \subseteq \mathscr{M}(u, v)$, the injective mapping $\mathbf{m} \mapsto \lambda(\mathbf{m})$ restricts to $\mathscr{M}^{J}(u, v)$. We write $\left(a_{1}, \ldots, a_{k}\right) \prec\left(b_{1}, \ldots, b_{k}\right)$ for the antilexicographic order relation of distinct integer strings. The following lemma is known (see Lemma 2.7.4 in [2]).

Lemma 2.7 1. There is a unique chain $\mathbf{m}_{0} \in \mathscr{M}^{J}(u, v)$ such that $\lambda\left(\mathbf{m}_{0}\right)$ is decreasing $^{1}$ (meaning that $\left.\lambda_{1}\left(\mathbf{m}_{0}\right)>\lambda_{2}\left(\mathbf{m}_{0}\right)>\ldots>\lambda_{k}\left(\mathbf{m}_{0}\right)\right)$.

2. $\lambda\left(\mathbf{m}_{0}\right) \prec \lambda(\mathbf{m})$ for all $\mathbf{m} \neq \mathbf{m}_{0}$ in $\mathscr{M}^{J}(u, v)$.

We now recall what the Hecke algebra $\mathscr{H}$ of a Coxeter group $W$ is. Let $A:=$ $\mathbb{Z}\left[q^{-1 / 2}, q^{1 / 2}\right]$ be the ring of Laurent polynomials in the indeterminate $q^{1 / 2}$. The Hecke algebra $\mathscr{H}$ is the free $A$-module generated by the set $\left\{T_{w} \mid w \in W\right\}$ with the product

$$
T_{w} T_{s}= \begin{cases}T_{w s}, & \text { if } s \notin D_{R}(w), \\ q T_{w s}+(q-1) T_{w}, & \text { otherwise, }\end{cases}
$$

for all $w \in W$ and $s \in S$. For $s \in S$ the inverse of the generator $T_{S}$ is

$$
T_{s}^{-1}=\left(q^{-1}-1\right) T_{e}+q^{-1} T_{s}
$$

and this can be used to invert all the elements $T_{w}$, where $w \in W$. There is an involution $\iota$ on $\mathscr{H}$, as defined in [9], such that

$$
\iota\left(q^{1 / 2}\right)=q^{-1 / 2}, \iota\left(T_{w}\right)=T_{w^{-1}}^{-1},
$$

for all $w \in W$. Moreover this map is a ring automorphism (see [8], Section 7.7), i.e.

$$
\iota\left(T_{v} T_{w}\right)=\iota\left(T_{v}\right) \iota\left(T_{w}\right) \forall v, w \in W .
$$

\footnotetext{
1 This is consistent with our definition of $W^{J}$. The statement for the quotient ${ }^{J} W:=$ $\{w \in W \mid w s>w \forall s \in J\}$ says that the string $\lambda\left(\mathbf{m}_{0}\right)$ is increasing.
} 
Expanding the element $\iota\left(T_{w}\right)$ in terms of the basis $\left\{T_{w} \mid w \in W\right\}$ and considering an $\iota$-invariant basis $\left\{C_{w}\right\}_{w \in W}$ of $\mathscr{H}$, Kazhdan and Lusztig have defined two families of polynomials $\left\{R_{y, w}\right\}_{y, w \in W} \subseteq \mathbb{Z}[q]$ and $\left\{P_{y, w}\right\}_{y, w \in W} \subseteq \mathbb{Z}[q]$ by

$$
\begin{aligned}
\iota\left(T_{w}\right) & =q^{-\ell(w)} \sum_{y \leqslant w}(-1)^{\ell(y, w)} R_{y, w}(q) T_{y}, \\
C_{w} & =q^{\frac{\ell(w)}{2}} \sum_{y \leqslant w}(-1)^{\ell(y, w)} q^{-\ell(y)} P_{y, w}\left(q^{-1}\right) T_{y},
\end{aligned}
$$

for all $w \in W$.

The degree, the leading coefficient and the constant term of the R-polynomials are known (see Proposition 5.1.3 in [2]).

Proposition 2.8 Let $u, v \in W, u \leqslant v$. Then, $R_{u, v}$ is a monic polynomial of degree $\ell(u, v)$ and constant term $(-1)^{\ell(u, v)}$.

The constant term of the Kazhdan-Lusztig polynomials is known (see Proposition 5.1.5 in [2]): if $u, v \in W$ and $u \leqslant v$, then

$$
P_{u, v}(0)=1
$$

A generalization of these polynomials in a parabolic setting was given by Deodhar in [6]. He defined two double families of polynomials $\left\{R_{v, w}^{J, x}\right\}_{v, w \in W^{J}} \subseteq \mathbb{Z}[q]$ and $\left\{P_{v, w}^{J, x}\right\}_{v, w \in W^{J}} \subseteq \mathbb{Z}[q]$, where $x \in\{-1, q\}, J \subseteq S$. These families satisfy the following theorems (see [6]):

Theorem 2.9 Let $(W, S)$ be a Coxeter system, and $J \subseteq S$. Then, for each $x \in$ $\{-1, q\}$, there is a unique family of polynomials $\left\{R_{v, w}^{J, x}\right\}_{v, w \in W^{J}} \subseteq \mathbb{Z}[q]$ such that, for all $v, w \in W^{J}$ :

1. $R_{v, w}^{J, x}=0$ if $v \nless w_{\text {; }}$

2. $R_{w, w}^{J, x}=1$;

3. if $v<w$ and $s \in D_{R}(w)$ then

$$
R_{v, w}^{J, x}= \begin{cases}R_{v, x, w s}^{J, x}, & \text { if } s \in D_{R}(v), \\ q R_{v s, w s}^{J, x}+(q-1) R_{v, w s}^{J, x}, & \text { if } s \notin D_{R}(v) \text { and } v s \in W^{J} \\ (q-1-x) R_{v, w s}^{J, x}, & \text { if } s \notin D_{R}(v) \text { and } v s \notin W^{J}\end{cases}
$$

Theorem 2.10 Let $(W, S)$ be a Coxeter system, and $J \subseteq S$. Then, for each $x \in$ $\{-1, q\}$, there is a unique family of polynomials $\left\{P_{v, w}^{J, x}\right\}_{v, w \in W^{J}} \subseteq \mathbb{Z}[q]$ such that, for all $v, w \in W^{J}$ :

1. $P_{v, w}^{J, x}=0$ if $v \nless w$;

2. $P_{w, w}^{J, x}=1$;

3. $\operatorname{deg}\left(P_{v, w}^{J, x}\right) \leqslant \frac{\ell(v, w)-1}{2}$, if $v<w$ 
4.

$$
q^{\ell(v, w)} P_{v, w}^{J, x}\left(q^{-1}\right)=\sum_{z \in[v, w]^{J}} R_{v, z}^{J, x}(q) P_{z, w}^{J, x}(q),
$$

if $v \leqslant w$.

The polynomials $R_{v, w}^{J, x}(q)$ and $P_{v, w}^{J, x}(q)$ are called the parabolic R-polynomials and parabolic Kazhdan-Lusztig polynomials (respectively) of $W^{J}$ of type $x$. They generalize the ordinary R-polynomials and Kazhdan-Lusztig polynomials of $W$, which appear in the expansions (11) and (12), since they satisfy the following relations:

$$
\begin{aligned}
& R_{v, w}^{\varnothing, x}=R_{v, w}, \\
& P_{v, w}^{\varnothing, x}=P_{v, w},
\end{aligned}
$$

for all $x \in\{-1, q\}$ and $v, w \in W$. For $u, v \in W^{J}$, the parabolic $R$-polynomials are related to the ordinary ones by the following equality (see Proposition 2.12. in [6]):

$$
R_{u, v}^{J, x}=\sum_{w \in W_{J}}(-x)^{\ell(w)} R_{w u, v} .
$$

Remark 3.8. of [6] points out the following relation between the parabolic KazhdanLusztig polynomials and the ordinary ones for $x=q$ :

$$
P_{u, v}^{J, q}=\sum_{w \in W_{J}}(-1)^{\ell(w)} P_{w u, v},
$$

for all $u, v \in W^{J}$. The following is another result which will be useful in the sequel (see Corollary 2.2 in [7]).

Proposition 2.11 Let $(W, S)$ be a Coxeter system, and $J \subseteq S$. Then

$$
(-q)^{\ell(u, v)} R_{u, v}^{J, x}\left(q^{-1}\right)=R_{u, v}^{J, q-1-x}(q)
$$

for all $u, v \in W^{J}$, and $x \in\{-1, q\}$.

We end this section by recalling some notions from the theory of $P$-kernels in a poset $(P, \leqslant)$. For further details see [13], and [4] for applications to parabolic KazhdanLusztig theory. We follow Chapter 3 of [14] for notation and terminology concerning posets. Given a poset $P$, we let $\operatorname{Int}(P):=\{(x, y) \in P \times P \mid x \leqslant y\}$; we say that $P$ is locally finite if $|[x, y]|<\infty$ for all $(x, y) \in \operatorname{Int}(P)$. Given a locally finite poset and a commutative ring $R$ the incidence algebra of $P$ with coefficients in $R$ is denoted $I(P ; R)$.

Let $P$ be a locally finite poset. We say that a function $\rho: \operatorname{Int}(P) \rightarrow \mathbb{N}$ is a weak rank function for $P$ if it has the following properties:

1. if $u<v$ then $\rho(u, v)>0$; 
2. if $u \leqslant a \leqslant v$ then $\rho(u, v)=\rho(u, a)+\rho(a, v)$.

Note that a weak rank function always exists. Following Sect. 6 of [13], given a locally finite poset $P$ and a weak rank function $\rho$ for $P$, we let

$$
\begin{aligned}
\tilde{I}(P) & :=\{f \in I(P ; \mathbb{R}[q]) \mid \operatorname{deg}(f(x, y)) \leqslant \rho(x, y) \text { for all }(x, y) \in \operatorname{Int}(P)\} ; \\
I_{1 / 2}(P) & :=\left\{f \in \tilde{I}(P) \mid \operatorname{deg}(f(x, y)) \leqslant \frac{\rho(x, y)-1}{2} \text { for } x<y, \text { and } f(x, x)=1\right\} .
\end{aligned}
$$

Given $f \in \tilde{I}(P)$ we let

$$
\bar{f}(x, y)(q):=q^{\rho(x, y)} f(x, y)\left(q^{-1}\right),
$$

for all $x, y \in P, x \leqslant y$. Given an invertible element $f \in \tilde{I}(P)$, Lemma 6.1. in [13] asserts that

$$
(\bar{f})^{-1}=\bar{f}^{-1} .
$$

Recall (see Definition 6.2 in [13]) that an element $\mathscr{K} \in I(P ; \mathbb{R}[q])$ is called a $P$-kernel if $\mathscr{K}(x, x)=1$ for all $x \in P$, and there exists an element $f \in \tilde{I}(P)$ such that

1. $f$ is invertible in $I(P ; \mathbb{R}[q])$;

2. $\mathscr{K} f=\bar{f}$.

An element $f \in \tilde{I}(P)$ satisfying (2) above is called $\mathscr{K}$-totally acceptable (Definition 6.2 of [13] $)^{2}$. The next result, which is a generalization of Corollary 6.7 in [13], appears in [4] as Theorem 6.2.

Theorem 2.12 Let $P$ be a locally finite poset and $\mathscr{K} \in I(P ; \mathbb{R}[q])$ be a P-kernel. Then there exists a unique element $\gamma \in I_{1 / 2}(P)$ such that $\mathscr{K} \gamma=\bar{\gamma}$.

We call the element $\gamma$, whose existence and uniqueness are guaranteed by the preceding theorem, the Kazhdan-Lusztig-Stanley function (or KLS-function, for short) of $P$ relative to $\mathscr{K}$.

Let $f \in \tilde{I}(P)$ and $i$ be a positive integer. For a multichain $a_{0} \leqslant a_{1} \leqslant \ldots \leqslant a_{i}$ in $P$ we define a polynomial $f_{a_{0}, \ldots, a_{i}} \in \mathbb{R}[q]$ inductively as follows. We let

$$
f_{a_{0}, \ldots, a_{i}}(q):=f_{a_{0}, a_{1}}(q) U_{\left(\rho\left(a_{1}, a_{i}\right)+1\right) / 2}\left(q^{\rho\left(a_{1}, a_{i}\right)} f_{a_{1}, \ldots, a_{i}}\left(q^{-1}\right)\right)
$$

if $i \geqslant 2$, and

$$
f_{a_{0}, \ldots, a_{i}}:=f\left(a_{0}, a_{1}\right)
$$

if $i=1$, where the operator $U_{j}: \mathbb{R}[q] \rightarrow \mathbb{R}[q]$ is defined, for $j \in \mathbb{Q}$, by

$$
U_{j}\left(\sum_{i \geqslant 0} a_{i} q^{i}\right):=\sum_{i \geqslant j} a_{i} q^{i}
$$

\footnotetext{
2 These definitions are a little different from those in [13]: Stanley uses " $f \mathscr{K}$ " instead of " $\mathscr{K} f$ ". This choice is convenient for our purposes and does not affect the validity of the results in the sequel.
} 
We then have the following result (see Corollary 6.5 in [4]):

Theorem 2.13 Let $P$ be a locally finite poset, $\mathscr{K}$, be a $P$-kernel and $\gamma$ be the KLSfunction of $P$ relative to $\mathscr{K}$. Then, for all $u, v \in P, u \leqslant v$,

$$
\gamma(u, v)=\sum_{\mathscr{C} \in \mathbf{M}(u, v)}(\overline{\mathscr{K}})_{\mathscr{C}},
$$

where $\mathbf{M}(u, v)$ denotes the set of all multichains in $P$ from $u$ to $v$.

\section{The annihilator of $m_{e}^{J, x}$}

In order to define the parabolic $R$-polynomials in a Coxeter system $(W, S)$, in [6] Deodhar introduced for any $J \subseteq S$ a free $A$-module $M^{J}$ generated by $W^{J}$, i.e. $M^{J}=\operatorname{span}_{A}\left\{m_{v}^{J} \mid v \in W^{J}\right\}$, and two surjective maps $\phi^{J, x}: \mathscr{H} \rightarrow M^{J}$ defined by $\phi^{J, x}\left(T_{w}\right)=x^{\ell\left(w_{J}\right)} m_{w^{J}}^{J}$, where $x \in\{-1, q\}$. He constructed an action of the Hecke algebra $\mathscr{H}$ on $M^{J}$, making this module an $\mathscr{H}$-module, and the map $\phi^{J, x}$ an epimorphism of $\mathscr{H}$-modules for both $x \in\{-1, q\}$. In our conventions we are speaking about right $\mathscr{H}$-modules. Explicitly, the two actions of the Hecke algebra on $M^{J}$ are given by $m_{v}^{J} T_{w}=\phi^{J, x}\left(T_{v} T_{w}\right)$. To distinguish the two possible $\mathscr{H}$-modules for $x \in\{-1, q\}$, we write $M^{J, x}$ and their elements as $m_{v}^{J, x}$, for $v \in W^{J}$. Obviously $M^{J, q}=M^{J,-1}=M^{J}$ as $A$-modules. There is an involution $\iota^{J, x}$ on $M^{J, x}$, analogous to the one defined on $\mathscr{H}$ in Eq. (9), such that

$$
\iota^{J, x}\left(\phi^{J, x}\left(T_{w}\right)\right)=\phi^{J, x}\left(\iota\left(T_{w}\right)\right),
$$

for every $w \in W$ (see [6], Sect. 3).

The $\mathscr{H}$-modules $M^{J, x}$ can be described also in another way. In fact these modules are principal because $m_{v}^{J, x}=m_{e}^{J, x} T_{v}$, for all $v \in W^{J}$. Then $M^{J, x}$ is isomorphic as a right $\mathscr{H}$-module to $\mathscr{H} / \operatorname{ann}_{e}^{J, x}$, where the right ideal $\operatorname{ann}_{e}^{J, x}:=$ $\left\{a \in \mathscr{H} \mid m_{e}^{J, x} a=0\right\}=\operatorname{ker}\left(\phi^{J, x}\right)$ is the annihilator of $m_{e}^{J, x}$. For every $J \subseteq S, v \in$ $W \backslash W^{J}$ and $x \in\{-1, q\}$ we define an element $b_{v}^{J, x} \in \operatorname{ann}_{e}^{J, x}$ by

$$
b_{v}^{J, x}:=x^{\ell\left(v_{J}\right)} T_{v^{J}}-T_{v} .
$$

Observe that $b_{v}^{J, x}=0$ if and only if $v \in W^{J}$.

Definition 3.1 For $J \subseteq S, x \in\{-1, q\}$ and any sequence $\left\{a_{w}\right\}_{w \in W} \subseteq A$, we define

$$
a_{v}^{J, x}:=\sum_{w \in W_{J}} x^{\ell(w)} a_{w v},
$$

for all $v \in W^{J}$. 
Proposition 3.2 Let $a=\sum_{v \in W} a_{v} T_{v}$ be an element of $\mathscr{H}$, where $a_{v} \in$ A for each $v \in W$, and $J \subseteq S$. Then

$$
a=\sum_{v \in W^{J}} a_{v}^{J, x} T_{v}-\sum_{v \in W \backslash W^{J}} a_{v} b_{v}^{J, x} .
$$

Moreover $a \in \operatorname{ann}_{e}^{J, x}$ if and only if $a_{v}^{J, x}=0$, for all $v \in W^{J}$. In particular, when $a \in \operatorname{ann}_{e}^{J, x}$,

$$
a=-\sum_{v \in W \backslash W^{J}} a_{v} b_{v}^{J, x}
$$

Proof It is an easy calculation based on the fact that, if $v \in W \backslash W^{J}$, then $T_{v}=$ $x^{\ell\left(v_{J}\right)} T_{v^{J}}-b_{v}^{J, x}$.

Corollary 3.3 The set $\mathscr{B}^{J, x}:=\left\{b_{w}^{J, x} \mid w \in W \backslash W^{J}\right\}$ is an A-basis of $\operatorname{ann}_{e}^{J, x}$, for every $J \subseteq S, x \in\{-1, q\}$.

Proof The elements of $\mathscr{B}^{J, x}$ are $A$-linearly independent. In fact, by Proposition 3.2,

$$
\sum_{v \in W \backslash W^{J}} a_{v} b_{v}^{J, x}=\sum_{v \in W^{J}}\left(a_{v}^{J, x}-a_{v}\right) T_{v}-\sum_{v \in W \backslash W^{J}} a_{v} T_{v},
$$

so $\sum_{v \in W \backslash W^{J}} a_{v} b_{v}^{J, x}=0$ implies $a_{v}=0$ for every $v \in W \backslash W^{J}$. Moreover Proposition 3.2 proves that the set $\mathscr{B}^{J, x}$ generates $\operatorname{ann}_{e}^{J, x}$.

We can complete the basis $\mathscr{B}^{J, x}$, defined in Corollary 3.3 , to obtain a basis of the Hecke algebra, by taking $\left\{T_{v} \mid v \in W^{J}\right\} \cup \mathscr{B}^{J, x}$ as a new basis.

The following proposition explores the structure of $\operatorname{ann}_{e}^{J, x}$ as a right $\mathscr{H}$-module.

Proposition 3.4 Let $s \in S, J \subseteq S, w \in W \backslash W^{J}$ and $x \in\{-1, q\}$. Then

$$
b_{w}^{J, x} T_{s}= \begin{cases}b_{w s}^{J, x}, & \text { if } s \notin D_{R}(w) \text { and } w^{J} s \in W^{J} ; \\ b_{w s}^{J, x}-x^{\ell\left(w_{J}\right)} b_{w^{J} s}^{J, x}, & \text { if } s \notin D_{R}(w) \text { and } w^{J} s \notin W^{J} ; \\ q b_{w s}^{J, x}+(q-1) b_{w}^{J, x} & \text { if } s \in D_{R}\left(w^{J}\right) ; \\ q b_{w s}^{J, x}+(q-1) b_{w}^{J, x}-x^{\ell\left(w_{J}\right)} b_{w^{J} s}^{J, x} & \text { if } s \in D_{R}(w) \backslash D_{R}\left(w^{J}\right) .\end{cases}
$$

Proof We have a few cases to distinguish.

1. If $s \notin D_{R}(w)$ and $w^{J} s \in W^{J}$, then $(w s)^{J}=w^{J} s$ and $(w s)_{J}=w_{J}$.

2. If $s \notin D_{R}(w)$ and $w^{J} s \notin W^{J}$ then $(w s)^{J}=w^{J}$ and $(w s)_{J}=w_{J} s^{\prime}$ for some $s^{\prime} \in J$, by Corollary 2.5. So

$$
b_{w}^{J, x} T_{s}=\left(x^{\ell\left(w_{J}\right)} T_{w^{J}}-T_{w}\right) T_{s}=x^{\ell\left(w_{J}\right)} T_{w^{J} s}-T_{w s} .
$$


Moreover $T_{w s}=x^{\ell\left((w s)_{J}\right)} T_{(w s)^{J}}-b_{w s}^{J, x}$, by the definition of $b_{w s}^{J, x}$. Therefore $T_{w s}=$ $x^{\ell\left(w_{J}\right)+1} T_{w^{J}}-b_{w s}^{J, x}$ and then

$$
b_{w}^{J, x} T_{s}=x^{\ell\left(w_{J}\right)} T_{w^{J}}-x^{\ell\left(w_{J}\right)+1} T_{w^{J}}+b_{w s}^{J, x}=b_{w s}^{J, x}-x^{\ell\left(w_{J}\right)} b_{w^{J} s}^{J, x} .
$$

3. If $s \in D_{R}\left(w^{J}\right)$, then $s \in D_{R}(w)$ and $(w s)^{J}=w^{J} s,(w s)_{J}=w_{J}$. So

$$
\begin{aligned}
b_{w}^{J, x} T_{s} & =\left(x^{\ell\left(w_{J}\right)} T_{w^{J}}-T_{w}\right) T_{s} \\
& =x^{\ell\left(w_{J}\right)}\left(q T_{w^{J}}+(q-1) T_{w^{J}}\right)-\left(q T_{w s}+(q-1) T_{w}\right) .
\end{aligned}
$$

4. If $s \in D_{R}(w) \backslash D_{R}\left(w^{J}\right)$ then, by Corollary 2.5 an $s^{\prime} \in J$ exists such that $w^{J} s=$ $s^{\prime} w^{J}$ and $w_{J} s^{\prime}<w_{J}$. So $(w s)_{J}=w_{J} s^{\prime}$ and $(w s)^{J}=w^{J}$. Then $b_{w}^{J, x} T_{s}=$ $x^{\ell\left(w_{J}\right)} T_{w^{J} s}-\left(q T_{w s}+(q-1) T_{w}\right)$. But

$$
T_{w^{J} s}=x^{\ell\left(\left(w^{J} s\right)_{J}\right)} T_{\left(w^{J} s\right)^{J}}-b_{w^{J} s}^{J, x}=x T_{w^{J}}-b_{w^{J} s}^{J, x}
$$

so

$$
b_{w}^{J, x} T_{s}=x^{\ell\left(w_{J}\right)+1} T_{w^{J}}-x^{\ell\left(w_{J}\right)} b_{w^{J} s}^{J, x}-q T_{w s}-(q-1) T_{w} .
$$

Now $T_{w s}=x^{\ell\left(w_{J}\right)-1} T_{w^{J}}-b_{w s}^{J, x}$ and then

$$
b_{w}^{J, x} T_{s}=x^{\ell\left(w_{J}\right)+1} T_{w^{J}}-x^{\ell\left(w_{J}\right)} b_{w^{J} s}^{J, x}-q x^{\ell\left(w_{J}\right)-1} T_{w^{J}}+q b_{w s}^{J, x}-(q-1) T_{w} .
$$

Since $x-q x^{-1}=q-1$, we have

$$
\begin{aligned}
b_{w}^{J, x} T_{s} & =x^{\ell\left(w_{J}\right)}(q-1) T_{w^{J}}-x^{\ell\left(w_{J}\right)} b_{w^{J}}^{J, x}+q b_{w s}^{J, x}-(q-1) T_{w} \\
& =q b_{w s}^{J, x}+(q-1) b_{w}^{J, x}-x^{\ell\left(w_{J}\right)} b_{w^{J} s}^{J, x} .
\end{aligned}
$$

Next we note that $\operatorname{ann}_{e}^{J, x}$ is an $\iota$-invariant right ideal of $\mathscr{H}$, where $\iota$ is the involution defined by Eq. (9).

Proposition 3.5 The right ideal $\operatorname{ann}_{e}^{J, x}$ is ı-invariant, for all $J \subseteq S, x \in\{-1, q\}$.

Proof In fact, if $a \in \operatorname{ann}_{e}^{J, x}, \phi^{J, x}(\iota(a))=\iota^{J, x}\left(\phi^{J, x}(a)\right)=0$, by Eq. (20).

Lusztig defined in [10] a map $\Phi: \mathscr{H} \rightarrow \mathscr{H}$ by $\Phi\left(q^{\frac{1}{2}}\right)=-q^{\frac{1}{2}}$ and $\Phi\left(T_{w}\right)=$ $(-q)^{\ell(w)} \iota\left(T_{w}\right)$. This map is an involution, and it commutes with $\iota$ (see [10], 5.1.15). We denote with $\Phi^{J, x}$ the restriction of $\Phi$ to $\operatorname{ann}_{e}^{J, x}$.

Proposition 3.6 If $J \subseteq S$, we have $\Phi\left(\operatorname{ann}_{e}^{J, x}\right)=\operatorname{ann}_{e}^{J, q-1-x}$ so $\Phi^{J, x}: \operatorname{ann}_{e}^{J, x} \rightarrow$ $\operatorname{ann}_{e}^{J, q-1-x}$ is a bijection; in particular

$$
\Phi\left(b_{w}^{J, x}\right)=(-q)^{\ell(w)} \iota\left(b_{w}^{J, q-1-x}\right)
$$


for all $w \in W \backslash W^{J}$.

Proof By definition

$$
\begin{aligned}
\Phi\left(b_{w}^{J, x}\right) & =\Phi\left(x^{\ell\left(w_{J}\right)} T_{w^{J}}-T_{w}\right) \\
& =x^{\ell\left(w_{J}\right)}(-q)^{\ell\left(w^{J}\right)} \iota\left(T_{w^{J}}\right)-(-q)^{\ell(w)} \iota\left(T_{w}\right) \\
& =(-q)^{\ell(w)}\left[(q-1-x)^{-\ell\left(w_{J}\right)} \iota\left(T_{w^{J}}\right)-\iota\left(T_{w}\right)\right] \\
& =(-q)^{\ell(w)} \iota\left(b_{w}^{J, q-1-x}\right) .
\end{aligned}
$$

\section{Polynomials}

Using the results of the previous section, we will define polynomials, indexed by pairs of elements of $W \backslash W^{J}$, which are analogous, for $W \backslash W^{J}$, to the parabolic $R$-polynomials defined by Deodhar for $W^{J}$.

Since, by Proposition 3.5, $\operatorname{ann}_{e}^{J, x}$ is $\iota$-invariant, we can express any element $\iota\left(b_{w}^{J, x}\right)$ in terms of the basis $\mathscr{B}^{J, x}$.

Definition 4.1 We define elements $\left\{Z_{y, w}^{J, x}\right\}_{y, w \in W \backslash W^{J}} \subseteq A$ by

$$
\iota\left(b_{w}^{J, x}\right)=q^{-\ell(w)} \sum_{y \in W \backslash W^{J}}(-1)^{\ell(y, w)} Z_{y, w}^{J, x} b_{y}^{J, x} .
$$

The next proposition gives a relation between the ordinary $R$-polynomials and the polynomials $Z^{J, x}$.

Proposition 4.2 For each $w \in W \backslash W^{J}$ and $x \in\{-1, q\}$, we have

$$
R_{v, w}-(q-1-x)^{\ell\left(w_{J}\right)} R_{v, w^{J}}= \begin{cases}Z_{v, w}^{J, x}, & \text { if } v \in W \backslash W^{J} \\ -\sum_{y \in W_{J} \backslash\{e\}}(-x)^{\ell(y)} Z_{y v, w}^{J, x}, & \text { if } v \in W^{J}\end{cases}
$$

for all $v \in W$.

Proof From the expansion (11) we find

$$
\begin{aligned}
\iota\left(b_{w}^{J, x}\right) & =x^{-\ell\left(w_{J}\right)} \iota\left(T_{w^{J}}\right)-\iota\left(T_{w}\right) \\
& =x^{-\ell\left(w_{J}\right)} q^{-\ell\left(w^{J}\right)} \sum_{y \leqslant w^{J}}(-1)^{\ell\left(y, w^{J}\right)} R_{y, w^{J}} T_{y}-q^{-\ell(w)} \sum_{y \leqslant w}(-1)^{\ell(y, w)} R_{y, w} T_{y} \\
& =\sum_{y \in W}\left((-1)^{\ell\left(y, w^{J}\right)} x^{-\ell\left(w_{J}\right)} q^{-\ell\left(w^{J}\right)} R_{y, w^{J}}-(-1)^{\ell(y, w)} q^{-\ell(w)} R_{y, w}\right) T_{y} .
\end{aligned}
$$


On the other hand, by Definition 4.1,

$$
\begin{aligned}
\iota\left(b_{w}^{J, x}\right)= & q^{-\ell(w)} \sum_{y \in W \backslash W^{J}}(-1)^{\ell(y, w)} Z_{y, w}^{J, x} b_{y}^{J, x} \\
= & q^{-\ell(w)} \sum_{y \in W \backslash W^{J}}(-1)^{\ell(y, w)} Z_{y, w}^{J, x}\left(x^{\ell\left(y_{J}\right)} T_{y}-T_{y}\right) \\
= & q^{-\ell(w)} \sum_{y \in W^{J}}(-1)^{\ell(y, w)}\left(\sum_{u \in W_{J} \backslash\{e\}}(-x)^{\ell(u)} Z_{u y, w}^{J, x}\right) T_{y} \\
& -q^{-\ell(w)} \sum_{y \in W \backslash W^{J}}(-1)^{\ell(y, w)} Z_{y, w}^{J, x} T_{y},
\end{aligned}
$$

by (21). Comparing the coefficients the result follows.

Corollary 4.3 Let $(W, S)$ be a Coxeter system, and $J \subseteq S$; then

$$
Z_{v, w}^{J, x}=R_{v, w}-(q-1-x)^{\ell\left(w_{J}\right)} R_{v, w^{J}},
$$

for all $v, w \in W \backslash W^{J}$ and $x \in\{-1, q\}$.

Remark 4.4 Let $I \subseteq J \subseteq S$, so $W^{J} \subseteq W^{I}$. We can generalize the results of this paper considering the morphisms of $\mathscr{H}$-modules $\phi^{I, J, x}: M^{I, x} \rightarrow M^{J, x}$ defined by

$$
\phi^{I, J, x}\left(m_{v}^{I, x}\right)=x^{\ell\left(v_{J}\right)} m_{v^{J}}^{J, x},
$$

for every $v \in W^{I}$ (see Sect. 5 of [6]). The kernels of these morphisms have an $A$ basis indexed by the set $W^{I} \backslash W^{J}$; moreover they are invariant under the involutions $\iota^{x}$ defined on $M^{I, x}$, for each $x \in\{-1, q\}$. This leads to a family of polynomials $\left\{Z_{v, w}^{I, J, x}\right\}_{v, w \in W^{I} \backslash W^{J}}$ such that

$$
Z_{v, w}^{I, J, x}=R_{v, w}^{I, x}-(q-1-x)^{\ell\left(w_{J}\right)} R_{v, w^{J}}^{I, x},
$$

for all $v, w \in W^{I} \backslash W^{J}$.

Example $4.5 \operatorname{In}\left(A_{5}, S\right)$, where $S=\left\{s_{1}, s_{2}, s_{3}, s_{4}, s_{5}\right\}$, the permutations $v=324156$ and $w=546132$ are in $W \backslash W^{S \backslash\left\{s_{3}\right\}}$ and $v<w$. Moreover $w^{J}=456123, \ell\left(w_{J}\right)=2$ and $v<w^{J}$. So, by Corollary 4.3,

$$
\begin{aligned}
Z_{v, w}^{J, q} & =R_{324156,546132}-R_{324156,456123}=q^{7}-4 q^{6}+6 q^{5}-4 q^{4}+q^{3}, \\
Z_{v, w}^{J,-1} & =R_{324156,546132-q^{2} R_{324156,456123}=-q^{4}+4 q^{3}-6 q^{2}+4 q-1 .} .
\end{aligned}
$$

Example 4.6 Take $J=\{s\}$. Then $W \backslash W^{\{s\}}=s W^{\{s\}}$, so $W \backslash W^{\{s\}} \simeq W^{\{s\}}$ as posets. Moreover, if $u, v \in W^{\{s\}}$,

$$
Z_{s u, s v}^{\{s\}, q-1-x}=R_{s u, s v}-x R_{s u, v}=R_{u, v}-x R_{s u, v}=R_{u, v}^{\{s\}, x}
$$


because $R_{s u, s v}=R_{u, v}$. The last equality follows from Eq. (16).

There are some immediate corollaries to Proposition 4.2, whose proof follows from the properties of the $R$-polynomials stated in Proposition 2.8 and in Theorem 2.9 (taking $J=\varnothing$ ).

Corollary 4.7 If $v, w \in W \backslash W^{J}$ and $v \nless w$, then $Z_{v, w}^{J, x}=0$ and $Z_{w, w}^{J, x}=1$.

Corollary 4.8 If $v, w \in W \backslash W^{J}$ and $v \leqslant w$, then $Z_{v, w}^{J, q}$ is a monic polynomial of degree $\ell(v, w)$.

Proof This follows from Corollary 4.3 and Proposition 2.8, since $w \notin W^{J}$ and $\operatorname{deg}\left(R_{v, w}\right)=\ell(v, w)>\ell\left(v, w^{J}\right) \geqslant \operatorname{deg}\left(R_{v, w^{J}}\right)$.

Corollary 4.9 If $v, w \in W \backslash W^{J}$ and $v \leqslant w$, then $\operatorname{deg}\left(Z_{v, w}^{J,-1}\right) \leqslant \ell(v, w)$. Moreover $\operatorname{deg}\left(Z_{v, w}^{J,-1}\right)=\ell(v, w)$ if and only if $v \nless w^{J}$.

Proof This follows from Corollary 4.3 and Proposition 2.8, since $w \notin W^{J}$, and if $v<w^{J}$, we have $\operatorname{deg}\left(R_{v, w}\right)=\ell(v, w)=\ell\left(w_{J}\right)+\ell\left(v, w^{J}\right)=\operatorname{deg}\left(q^{\ell\left(w_{J}\right)} R_{v, w^{J}}\right)$.

Remark 4.10 It would be interesting to compute the degree of $Z_{v, w}^{J,-1}$. It depends on the maximal $k \in \mathbb{N}$ such that $\ell\left(w_{J}\right) \leqslant k<\ell(v, w)$ and for which the coefficients $\left[q^{k}\right]\left(R_{v, w}\right)$ and $\left[q^{k-\ell\left(w_{J}\right)}\right]\left(R_{v, w^{J}}\right)$ are different or, equivalently (by Proposition 2.11), such that the coefficients $\left[q^{\ell(v, w)-k}\right]\left(R_{v, w}\right)$ and $\left[q^{\ell\left(v, w^{J}\right)-k+\ell\left(w_{J}\right)}\right]\left((-1)^{\ell\left(w_{J}\right)} R_{v, w^{J}}\right)=$ $\left[q^{\ell(v, w)-k}\right]\left((-1)^{\ell\left(w_{J}\right)} R_{v, w^{J}}\right)$ are not equal.

Corollary 4.11 If $v, w \in W \backslash W^{J}$ and $v<w$, then $Z_{v, w}^{J,-1}(0)=(-1)^{\ell(v, w)}$ and

$$
Z_{v, w}^{J, q}(0)= \begin{cases}(-1)^{\ell(v, w)}, & \text { if } v \nless w^{J}, \\ 0, & \text { otherwise. }\end{cases}
$$

Proof Since $R_{v, w}(0)=(-1)^{\ell(v, w)}$, if $v<w^{J}$ we have that $Z_{v, w}^{J, x}(0)=(-1)^{\ell(v, w)}-$ $(-1)^{\ell\left(w_{J}\right)}(-1)^{\ell\left(v, w^{J}\right)}=0$. Otherwise $Z_{v, w}^{J, x}(0)=R_{v, w}(0)=(-1)^{\ell(v, w)}$, by Proposition 2.8.

The following duality result is the analogue, for the polynomials $Z_{v, w}^{J, x}$, of Proposition 2.11.

Corollary 4.12 Let $(W, S)$ be a Coxeter system, and $J \subseteq S$. Then

$$
(-q)^{\ell(v, w)} Z_{v, w}^{J, x}\left(q^{-1}\right)=Z_{v, w}^{J, q-1-x}(q)
$$

for all $v, w \in W \backslash W^{J}$, and $x \in\{-1, q\}$. 
Proof From Corollary 4.3 and Proposition 2.11 we have

$$
\begin{aligned}
(-q)^{\ell(v, w)} Z_{v, w}^{J, x}\left(q^{-1}\right) & =(-q)^{\ell(v, w)}\left(R_{v, w}\left(q^{-1}\right)-(q-1-x)^{-\ell\left(w_{J}\right)} R_{v, w^{J}}\left(q^{-1}\right)\right) \\
& =R_{v, w}(q)-(-q)^{\ell\left(w_{J}\right)}(q-1-x)^{-\ell\left(w_{J}\right)} R_{v, w^{J}}(q) \\
& =R_{v, w}(q)-x^{\ell\left(w_{J}\right)} R_{v, w^{J}}(q)=Z_{v, w}^{J, q-1-x}(q) .
\end{aligned}
$$

By Corollary 4.3 and the recursion for the $R$-polynomials in Theorem 2.9, we can find a recursion for the polynomials $Z^{J, x}$. Note that $D_{R}\left(w^{J}\right)=\varnothing$ implies $w \in W_{J}$.

Proposition 4.13 Let $v, w \in W \backslash W^{J}$ and $v \leqslant w$. Then, if $w^{J} s<w^{J}$ or $w s<w \in$ $W_{J}$,

$$
Z_{v, w}^{J, x}= \begin{cases}Z_{v s, w s}^{J, x}, & \text { if } v>v s \in W \backslash W^{J}, \\ (q-1-x)^{\ell(w)-1} \delta_{v, s} \delta_{w^{J}, e}-\hat{Z}_{v, w s}^{J, x}, & \text { if } v>v s \in W^{J}, \\ q Z_{v s, w s}^{J, x}+(q-1) Z_{v, w s}^{J, x}, & \text { if } v<v s,\end{cases}
$$

where $\hat{Z}_{v, w}^{J, x}:=\sum_{y \in W_{J} \backslash\{e\}}(-x)^{\ell(y)} Z_{y v^{J}, w}^{J, x}$, and $\delta_{x, y}$ is the Kronecker delta.

Proof Let $s \in D_{R}\left(w^{J}\right)$; then $s \in D_{R}(w),(w s)_{J}=w_{J}$ and $w^{J} s=(w s)^{J}$. If $s \notin D_{R}(v)$, we have, by Corollary 4.3 and the recursion of Theorem 2.9

$$
\begin{aligned}
Z_{v, w}^{J, x} & =R_{v, w}-(q-1-x)^{\ell\left(w_{J}\right)} R_{v, w^{J}} \\
& =q R_{v s, w s}+(q-1) R_{v, w s}-(q-1-x)^{\ell\left(w_{J}\right)}\left(q R_{v s, w^{J} s}+(q-1) R_{v, w^{J} s}\right) \\
& =q Z_{v s, w s}^{J, x}+(q-1) Z_{v, w s}^{J, x} .
\end{aligned}
$$

If $v>v s \in W \backslash W^{J}$, then

$$
Z_{v, w}^{J, x}=R_{v s, w s}-(q-1-x)^{\ell\left(w_{J}\right)} R_{v s, w^{J} s}=Z_{v s, w s}^{J, x} .
$$

If $v>v s \in W^{J}$, then by Proposition 4.2

$$
Z_{v, w}^{J, x}=R_{v s, w s}-(q-1-x)^{\ell\left(w_{J}\right)} R_{v s, w^{J} s}=-\sum_{y \in W_{J} \backslash\{e\}}(-x)^{\ell(y)} Z_{y v^{J}, w s}^{J, x},
$$

since, in this case, $v s=v^{J}$. Observe that, by the lifting property (Proposition 2.1) $v s=v^{J} \leqslant w^{J} s$, because, by Proposition 2.4, $v^{J}<w^{J}$.

If $w \in W_{J}$, we have $w^{J}=e$ and $v \in W_{J}$. Let $s \in D_{R}(w)$. If $s \notin D_{R}(v)$, then

$$
Z_{v, w}^{J, x}=R_{v, w}=q R_{v s, w s}+(q-1) R_{v, w s}=q Z_{v s, w s}^{J, x}+(q-1) Z_{v, w s}^{J, x},
$$

since $v \neq e$. 


$$
\begin{aligned}
& \text { If } v>v s \in W \backslash W^{J} \text {, then } \\
& \qquad Z_{v, w}^{J, x}=R_{v, w}-(q-1-x)^{\ell(w)} R_{v, e}=R_{v, w}=R_{v s, w s}=Z_{v s, w s}^{J, x} .
\end{aligned}
$$

If $v=s$ we have $v s=e \in W^{J}$ and, by Proposition 4.2,

$$
Z_{v, w}^{J, x}=R_{s, w}=R_{e, w s}=(q-1-x)^{\ell(w)-1}-\sum_{y \in W_{J} \backslash\{e\}}(-x)^{\ell(y)} Z_{y, w s}^{J, x} .
$$

Summarizing the previous results we obtain the following statement.

Theorem 4.14 Let $(W, S)$ be a Coxeter system, and $J \subseteq S$. Then, for each $x \in$ $\{-1, q\}$, there is a unique family of polynomials $\left\{Z_{v, w}^{J, x}\right\}_{v, w \in W \backslash W^{J}} \subseteq \mathbb{Z}[q]$ such that, for all $v, w \in W \backslash W^{J}$ :

1. $Z_{v, w}^{J, x}=0$ if $v \nless w$;

2. $Z_{w, w}^{J, x}=1$;

3. if $v<w, s \in S$ and $w^{J} s<w^{J}$ or $w s<w \in W_{J}$ then

$$
Z_{v, w}^{J, x}= \begin{cases}Z_{v s, w s}^{J, x}, & \text { if } v>v s \in W \backslash W^{J}, \\ (q-1-x)^{\ell(w)-1} \delta_{v, s} \delta_{w^{J}, e}-\hat{Z}_{v, w s}^{J, x}, & \text { if } v>v s \in W^{J} \\ q Z_{v s, w s}^{J, x}+(q-1) Z_{v, w s}^{J, x}, & \text { if } v<v s\end{cases}
$$

where $\hat{Z}_{v, w}^{J, x}:=\sum_{y \in W_{J} \backslash\{e\}}(-x)^{\ell(y)} Z_{y v^{J}, w}^{J, x}$ and $\delta_{x, y}$ is the Kronecker delta.

\section{The poset $W \backslash W^{J}$}

In this section we investigate the set $W \backslash W^{J}$ with the induced Bruhat order, which is the underlying poset of our previous discussions. This poset does not in general have a minimum, but, when the group $W$ is finite, it has a maximum: $w_{0}$, the maximum of $W$. The minimal elements of $W \backslash W^{J}$ are the elements of $J$. If $I, J \subseteq S$, then

$$
W \backslash W^{I \cup J}=\left(W \backslash W^{I}\right) \cup\left(W \backslash W^{J}\right),
$$

since $W^{I \cup J}=W^{I} \cap W^{J}$. Moreover $I \subseteq J \subseteq S$ implies $\left(W \backslash W^{I}\right) \subseteq\left(W \backslash W^{J}\right)$. By the Subword Property of the Bruhat order (see Theorem 2.2.2 in [2]) and the factorization $w=w_{J} w^{J}$ for all $w \in W$, we have a surjective immersion of posets, not invertible as poset morphism,

$$
\left(W_{J} \backslash\{e\}\right) \times W^{J} \hookrightarrow W \backslash W^{J},
$$

where on $\left(W_{J} \backslash\{e\}\right) \times W^{J}$ we consider the product order. 
Lemma 5.1 Let $J \subseteq S$ and $u, v \in W \backslash W^{J}$. Then $[u, v]^{\backslash J}=[u, v]$ if and only if $u \nless v^{J}$.

Proof If $u<v^{J}$, then $v^{J} \in[u, v]$ but $v^{J} \notin[u, v]^{\backslash J}$. If $[u, v]^{\backslash J} \neq[u, v]$, then there is a $w \in W^{J} \cap[u, v]$. By Proposition 2.4 we have $w \leqslant v^{J}$, so $u<w \leqslant v^{J}$.

Theorem 5.2 The set $W \backslash W^{J}$, with the ordering induced by the Bruhat order, is a graded poset with the length minus one as rank function.

Proof Let $u, v \in W \backslash W^{J}$ be such that $u<v$. The case $u \nless v^{J}$ is obvious since, by Lemma 5.1, $[u, v]^{\backslash J}=[u, v]$. Let $v=v_{J} v^{J}=s_{1} \ldots s_{k} t_{1} \ldots t_{h}$, where $s_{1} \ldots s_{k}$ is a reduced expression for $v_{J}$, and $t_{1} \ldots t_{h}$ is a reduced expression for $v^{J}$, and $k \geqslant 1$.

If $u<v^{J}$ and $s_{1} \notin D_{L}(u)$, then $u \in W^{\left\{s_{1}\right\}}, s_{1} v \in W^{\left\{s_{1}\right\}}$ and $u<v^{J} \leqslant s_{1} v$. In this case, by Theorem 2.2, there is a maximal chain $u=x_{0}<x_{1}<\ldots<x_{r}=s_{1} v$ such that $\ell\left(x_{j}\right)=\ell\left(x_{0}\right)+j$ for $0 \leqslant j \leqslant r$. Then there is a maximal chain $u=$ $x_{0}<s_{1} x_{0}<s_{1} x_{1}<\ldots<s_{1} x_{r}$ in $W \backslash W^{J}$ such that $\ell\left(s_{1} x_{j}\right)=\ell\left(x_{0}\right)+j+1$ for $0 \leqslant j \leqslant r$.

If $s_{1} \in D_{L}(u)$ then $s_{1} u \in W^{\left\{s_{1}\right\}}$ and $s_{1} u<u<v^{J} \leqslant s_{1} v$. In this case, by Theorem 2.2, we have a maximal chain $s_{1} u=x_{0}<x_{1}<\ldots<x_{r}=s_{1} v$ in $W^{\left\{s_{1}\right\}}$ such that $\ell\left(x_{j}\right)=\ell\left(x_{0}\right)+j$ for $0 \leqslant j \leqslant r$. Then there is a maximal chain $u=s_{1} x_{0}<s_{1} x_{1}<\ldots<s_{1} x_{r}$ in $W \backslash W^{J}$ such that $\ell\left(s_{1} x_{j}\right)=\ell\left(s_{1} x_{0}\right)+j$ for $0 \leqslant j \leqslant r$.

Let $\mathscr{M}^{\backslash J}(u, v)$ denote the set of maximal chains in the interval $[u, v]^{\backslash J}$. Since $\mathscr{M}^{\backslash J}(u, v) \subseteq \mathscr{M}(u, v)$, the injective mapping $\mathbf{m} \mapsto \lambda(\mathbf{m})$ restricts to $\mathscr{M}^{\backslash J}(u, v)$.

Theorem 5.3 Let $J \subseteq S$ and $u, v \in W \backslash W^{J}$. Then

1. there is a unique chain $\mathbf{m}_{0} \in \mathscr{M}^{\backslash J}(u, v)$ such that $\lambda\left(\mathbf{m}_{0}\right)$ is decreasing,

2. $\lambda\left(\mathbf{m}_{0}\right) \prec \lambda(\mathbf{m})$ for all $\mathbf{m} \neq \mathbf{m}_{0}$ in $\mathscr{M}^{\backslash J}(u, v)$.

Proof Let $v$ have a reduced expression as in the proof of Theorem 5.2. If $u \nless v^{J}$ then, by Lemma 5.1, $[u, v]^{\backslash J}=[u, v]$, and we can apply Lemma 2.7 with $J=\varnothing$. Otherwise, if $u<v^{J}$ and $s_{1} \notin D_{L}(u)$, then $u \in W^{\left\{s_{1}\right\}}, s_{1} v \in W^{\left\{s_{1}\right\}}$ and $u<v^{J} \leqslant$ $s_{1} v$. In this case, by Lemma 2.7, we have a unique chain $\mathbf{m} \in \mathscr{M}^{\left\{s_{1}\right\}}\left(u, s_{1} v\right)$ such that $\lambda\left(u=x_{0}<x_{1}<\ldots<x_{r}=s_{1} v\right)$ is decreasing. Then $\lambda\left(u=x_{0}<s_{1} x_{0}<s_{1} x_{1}<\right.$ $\left.\ldots<s_{1} x_{r}=v\right)$ is still decreasing.

If $s_{1} \in D_{L}(u)$, then $s_{1} u \in W^{\left\{s_{1}\right\}}$ and $s_{1} u<u<v^{J} \leqslant s_{1} v$. In this case, by Lemma 2.7, we have a unique chain $\mathbf{m} \in \mathscr{M}^{\left\{s_{1}\right\}}\left(s_{1} u, s_{1} v\right)$ such that $\lambda\left(s_{1} u=x_{0}<\right.$ $\left.x_{1}<\ldots<x_{r}=s_{1} v\right)$ is decreasing. Then $\lambda\left(u=s_{1} x_{0}<s_{1} x_{1}<\ldots<s_{1} x_{r}=v\right)$ is still decreasing.

The proof of the second point is analogous to the one of Lemma 2.7.4 in [2].

Let $u, v \in W \backslash W^{J}$ and $(u, v)^{\backslash J}:=\left\{z \in W \backslash W^{J} \mid u<z<v\right\}$.

Corollary 5.4 The order complex of $(u, v)^{\backslash J}$ is shellable. In particular, it is CohenMacaulay.

Moreover, by Lemma 5.1, we have the following statement. 
Corollary 5.5 The order complex of $(u, v)^{\backslash J}$ is PL homeomorphic to

1. the sphere $\mathbb{S}^{\ell(u, v)-2}$, if $u \nless v^{J}$;

2. the ball $\mathbb{B}^{\ell(u, v)-2}$, otherwise.

From these results we can deduce the Möbius function of the poset $W \backslash W^{J}$.

Corollary 5.6 The Möbius function of the poset $W \backslash W^{J}$ is

$$
\mu^{\backslash J}(u, v)= \begin{cases}(-1)^{\ell(u, v)} & \text { if } u \nless v^{J}, \\ 0, & \text { otherwise. }\end{cases}
$$

Equivalently,

$$
\mu^{\backslash J}(u, v)= \begin{cases}(-1)^{\ell(u, v)} & \text { if }[u, v]^{\backslash J}=[u, v], \\ 0, & \text { otherwise. }\end{cases}
$$

\section{Parabolic polynomials for $W \backslash W^{J}$}

In this section, using Proposition 3.5, we extend the parabolic $R$-polynomials $R_{u, v}^{J, x}$ and Kazhdan-Lusztig polynomials $P_{u, v}^{J, x}$ defined by Deodhar to the case $v \in W \backslash W^{J}$. For the parabolic $R$-polynomials we consider the sum in the right-hand side of Eq. (16).

Definition 6.1 For all $u, v \in W, J \subseteq S$ and $x \in\{-1, q\}$ we define

$$
R_{u, v}^{J, x}:=\sum_{w \in W_{J}}(-x)^{\ell(w)} R_{w u, v} .
$$

The next two results show that the sum in the previous definition has an expression in terms of the parabolic $R$-polynomials.

Proposition 6.2 If $u \in W^{J}$, then

$$
R_{u, v}^{J, x}=(q-1-x)^{\ell\left(v_{J}\right)} R_{u, v^{J}}^{J, x}
$$

for all $v \in W, x \in\{-1, q\}$.

Proof By Proposition $3.5 \iota\left(x^{\ell\left(v_{J}\right)} T_{v^{J}}-T_{v}\right) \in \operatorname{ann}_{e}^{J, x}$, so, by the expansion (11) and by Theorem 2.9,

$$
\begin{aligned}
& x^{-\ell\left(v_{J}\right)} q^{-\ell\left(v^{J}\right)} \sum_{y \leqslant v^{J}}(-1)^{\ell\left(y, v^{J}\right)} R_{y, v^{J}} T_{y}-q^{-\ell(v)} \sum_{y \leqslant v}(-1)^{\ell(y, v)} R_{y, v} T_{y}= \\
&=(-q)^{-\ell\left(v^{J}\right)} \sum_{y \in W}(-1)^{\ell(y)}\left(x^{-\ell\left(v_{J}\right)} R_{y, v^{J}}-(-q)^{-\ell\left(v_{J}\right)} R_{y, v}\right) T_{y} \in \operatorname{ann}_{e}^{J, x} .
\end{aligned}
$$


By Proposition 3.2 we have, for $y \in W^{J}$,

$$
x^{-\ell\left(v_{J}\right)} \sum_{w \in W_{J}}(-x)^{\ell(w)} R_{w y, v^{J}}-(-q)^{-\ell\left(v_{J}\right)} \sum_{w \in W_{J}}(-x)^{\ell(w)} R_{w y, v}=0 .
$$

Therefore

$$
\sum_{w \in W_{J}}(-x)^{\ell(w)} R_{w y, v}=\left(-\frac{q}{x}\right)^{\ell\left(v_{J}\right)} \sum_{w \in W_{J}}(-x)^{\ell(w)} R_{w y, v^{J}}
$$

and the result follows from equality (16).

Proposition 6.3 Let $J \subseteq S$, then

$$
R_{u, v}^{J,-1}=q^{\ell\left(v_{J}\right)} R_{u^{J}, v^{J}}^{J,-1},
$$

for all $u, v \in W$.

Proof Note that

$$
\sum_{w \in W_{J}} R_{w u, v}=\sum_{w \in W_{J}} R_{w u_{J} u^{J}, v}=\sum_{w \in W_{J}} R_{w u^{J}, v}=q^{\ell\left(v_{J}\right)} R_{u^{J}, v^{J}}^{J,-1}
$$

by Proposition 6.2 .

Corollary 6.4 We have

$$
\sum_{z \leqslant v}(-x)^{\ell(z)} R_{z, v}=(q-1-x)^{\ell(v)}
$$

for every $v \in W$ and $x \in\{-1, q\}$.

Proof Take $J=S$ and $u=e$ in Proposition 6.2.

Remark 6.5 The result of Corollary 6.4 for $x=-1$ was already noted in [12], Eq. (3.5). It could be proved by induction on $\ell(v)$ (see also Proposition 2.2.6. in [11]).

Remark 6.6 If $v \in W$ is an element such that $P_{u, v}=1$ for all $u \leqslant v$ we find, summing over the interval $[e, v]$ both sides of the identity in Corollary 6.4 with $x=-1$, that the Poincaré polynomial $W_{v}(q)$ of the interval $[e, v]$, where $W_{v}(q):=\sum_{u \leqslant v} q^{\ell(u)}$, is a reciprocal polynomial, i.e. $q^{\ell(v)} W_{v}\left(q^{-1}\right)=W_{v}(q)$.

Let $\left\{Q_{u, v}\right\}_{u, v \in W} \subseteq \mathbb{Z}[q]$ be the inverse Kazhdan-Lusztig polynomials, i.e. $Q_{u, v}=(-1)^{\ell(u, v)} P_{u, v}^{-1}$ in the incidence algebra $I(W, \mathbb{Z}[q])$, for all $u, v \in W$ (see [2]). 
By (19) we have that, since the inverses of the $R$-polynomials are $R_{u, v}^{-1}=$ $(-1)^{\ell(u, v)} R_{u, v}$,

$$
q^{\ell(u, v)} Q_{u, v}\left(q^{-1}\right)=\sum_{z \in[u, v]} Q_{u, z} R_{z, v}
$$

for all $u, v \in W$ such that $u \leqslant v$.

The following result is probably known but, since we have not found it in literature, it could be useful to deduce it from Corollary 6.4.

Proposition 6.7 Let $(W, S)$ be a Coxeter system. Then $Q_{e, v}=1$ for every $v \in W$.

Proof We proceed by induction on $\ell(v)$. If $\ell(v)=0$ the result is obvious. Let $\ell(v)>1$. Then, by Corollary 6.4,

$$
\begin{aligned}
q^{\ell(v)} Q_{e, v}\left(q^{-1}\right) & =\sum_{z \in[e, v]} Q_{e, z} R_{z, v} \\
& =\sum_{z \in[e, v)} Q_{e, z} R_{z, v}+Q_{e, v}=\sum_{z \in[e, v)} R_{z, v}+Q_{e, v} \\
& =q^{\ell(v)}-R_{v, v}+Q_{e, v}
\end{aligned}
$$

therefore $q^{\ell(v)} Q_{e, v}\left(q^{-1}\right)-Q_{e, v}=q^{\ell(v)}-1$ and then $Q_{e, v}=1$.

Now, after the extension of the parabolic $R$-polynomials, we extend the parabolic Kazhdan-Lusztig polynomials. Since equality (17) is well defined for all $u, v \in W$, the following definition is natural.

Definition 6.8 For all $u, v \in W, J \subseteq S$ and $x \in\{-1, q\}$ we define

$$
\hat{P}_{u, v}^{J, x}:=\sum_{w \in W_{J}}(q-1-x)^{\ell(w)} P_{w u, v} .
$$

Remark 6.9 Observe that, in general, $\operatorname{deg}\left(\hat{P}_{u, v}^{J,-1}\right) \nless \frac{\ell(u, v)-1}{2}$.

By Eq. (17) we have that $\hat{P}_{u, v}^{J, q}=P_{u, v}^{J, q}$ for all $u, v \in W^{J}$. On the contrary, in general we have $\hat{P}_{u, v}^{J,-1} \neq P_{u, v}^{J,-1}$, for $u, v \in W^{J}$; in fact, if $\left|W_{J}\right|<\infty, W_{J}(q)$ is its Poincaré polynomial, and $w_{0}(J)$ is the element of maximal length in $W_{J}$, it follows from the equality $P_{w u, w_{0}(J) v}=P_{w_{0}(J) u, w_{0}(J) v}$ for each $w \in W_{J}$ (see Proposition 5.1.8 in [2]) that $\hat{P}_{u, w_{0}(J) v}^{J,-1}=W_{J}(q) P_{w_{0}(J) u, w_{0}(J) v}=W_{J}(q) P_{u, v}^{J,-1}$ (see Proposition 3.4 in [6]). Then

$$
P_{u, v}^{J,-1}=\frac{1}{W_{J}(q)} \hat{P}_{u, w_{0}(J) v}^{J,-1}
$$

for all $u, v \in W^{J}$.

The next result shows that the polynomials $\hat{P}^{J, q}$ can be expressed in terms of the parabolic polynomials $P^{J, q}$. 
Proposition 6.10 Let $J \subseteq S$. Then

$$
\hat{P}_{u, v}^{J, q}=\left\{\begin{array}{l}
(-1)^{\ell\left(u_{J}\right)} P_{u^{J}, v}^{J, q}, \quad \text { if } v \in W^{J} \\
0, \text { otherwise. }
\end{array}\right.
$$

for all $u, v \in W$.

Proof If $v \notin W^{J}$ there exists $s \in D_{L}(v)$ such that $s \in J$ and then, since $P_{u, v}=P_{s u, v}$ (see Proposition 5.1.8 in [2]),

$$
\begin{aligned}
\sum_{w \in W_{J}}(-1)^{\ell(w)} P_{w u, v} & =\sum_{w \in W_{J}}(-1)^{\ell(w)} P_{s w u, v}=-\sum_{w \in W_{J}}(-1)^{\ell(s w)} P_{s w u, v} \\
& =-\sum_{w \in W_{J}}(-1)^{\ell(w)} P_{w u, v} .
\end{aligned}
$$

If $v \in W^{J}$ and $u \notin W^{J}$, consider the decomposition $u=u_{J} u^{J}$. So

$$
\begin{aligned}
\sum_{w \in W_{J}}(-1)^{\ell(w)} P_{w u, v} & =\sum_{w \in W_{J}}(-1)^{\ell(w)} P_{w u_{J} u^{J}, v} \\
& =(-1)^{\ell\left(u_{J}\right)} \sum_{w \in W_{J}}(-1)^{\ell\left(w u_{J}\right)} P_{w u_{J} u^{J}, v} \\
& =(-1)^{\ell\left(u_{J}\right)} \sum_{w \in W_{J}}(-1)^{\ell(w)} P_{w u^{J}, v}=(-1)^{\ell\left(u_{J}\right)} P_{u^{J}, v}^{J, q}
\end{aligned}
$$

by (17).

By the previous proposition we obtain the following corollary.

Corollary 6.11 Let $J \subseteq S, x \in\{-1, q\}$ and $v \notin W^{J}$. Then, for every $u \in W$,

$$
\hat{P}_{u, v}^{J, x} \in\langle q-x\rangle,
$$

where $\langle q-x\rangle$ is the ideal of the ring $\mathbb{Z}[q]$ generated by $q-x$.

Proof If $x=q$, we have $\hat{P}_{u, v}^{J, q}=0$ by Proposition 6.10 . Then -1 is a root of the polynomial $\hat{P}_{u, v}^{J,-1}$.

The following result, i.e. the expression in terms of the basis $\left\{T_{v} \mid v \in W^{J}\right\} \cup \mathscr{B}^{J, x}$ of the element $C_{w}$, defined in Eq. (12), is one of the main motivations of Definition 6.8.

Theorem 6.12 Let $v \in W$. The Kazhdan-Lusztig element $C_{v}$ is

$$
\begin{aligned}
C_{v}= & \sum_{y \in W^{J}}(-1)^{\ell(y, v)} q^{\frac{\ell(v)}{2}} q^{-\ell(y)} \hat{P}_{y, v}^{J, x}\left(q^{-1}\right) T_{y} \\
& -\sum_{y \in W \backslash W^{J}}(-1)^{\ell(y, v)} q^{\frac{\ell(v)}{2}} q^{-\ell(y)} P_{y, v}\left(q^{-1}\right) b_{y}^{J, x} .
\end{aligned}
$$


In particular, $C_{v} \in \operatorname{ann}_{e}^{J, q}$ if and only if $v \in W \backslash W^{J}$.

Proof The result follows, by (12), from Propositions 3.2 and 6.10.

Corollary 6.13 Let $J \subseteq S$ and $x \in\{-1, q\}$. Then

$$
q^{\ell(u, v)} \hat{P}_{u, v}^{J, x}\left(q^{-1}\right)=\sum_{y \in[u, v] \cap W^{J}} R_{u, y}^{J, x}(q) \hat{P}_{y, v}^{J, x}(q),
$$

for all $u \in W^{J}, v \in W$.

Proof Since $b_{y}^{J, x} \in \operatorname{ker}\left(\phi^{J, x}\right)$ for all $y \in W \backslash W^{J}$, by Theorem 6.12 we have

$$
\phi^{J, x}\left(C_{v}\right)=\sum_{y \in W^{J}}(-1)^{\ell(y, v)} q^{\frac{\ell(v)}{2}} q^{-\ell(y)} \hat{P}_{y, v}^{J, x}\left(q^{-1}\right) m_{y}^{J, x}
$$

Moreover, by (20), $\iota^{x}\left(\phi^{J, x}\left(C_{v}\right)\right)=\phi^{J, x}\left(\iota\left(C_{v}\right)\right)=\phi^{J, x}\left(C_{v}\right)$, and

$$
\iota^{x}\left(\phi^{J, x}\left(C_{v}\right)\right)=q^{-\frac{\ell(v)}{2}} \sum_{y \in W^{J}}(-1)^{\ell(y, v)}\left(\sum_{z \in W^{J}} R_{y, z}^{J, x}(q) \hat{P}_{z, v}^{J, x}(q)\right) m_{y}^{J, x}
$$

Comparing the coefficients the result follows.

The previous corollary shows, in particular, that $\hat{P}^{J,-1}$ is $R^{J,-1}$-totally acceptable for the poset $W^{J}$. By Remark 6.9, the result of Corollary 6.13 does not permit to compute the polynomials $\hat{P}^{J,-1}$ by recursion. We know that the $K L S$-function of $W^{J}$ relative to $R^{J,-1}$ is $P^{J,-1}$.

\section{$7\left(W \backslash W^{J}\right)$-kernels}

In this section we prove the existence of two $\left(W \backslash W^{J}\right)$-kernels, one for each $x \in$ $\{-1, q\}$. The weak rank function of this poset is $\ell(u, v)$.

We begin with $x=q$. The following result shows that $Z^{J, q} \in \tilde{I}\left(W \backslash W^{J}\right)$, where $Z^{J, q}(u, v):=Z_{u, v}^{J, q}$ for all $u, v \in W \backslash W^{J}$, is a $\left(W \backslash W^{J}\right)$-kernel and that the element $\tilde{P}^{J, q} \in I_{1 / 2}\left(W \backslash W^{J}\right)$, defined by $\tilde{P}^{J, q}(u, v):=P_{u, v}$, is the KLS-function of $W \backslash W^{J}$ relative to $Z^{J, q}$.

Theorem 7.1 If $u, v \in W \backslash W^{J}$, then

$$
q^{\ell(u, v)} P_{u, v}\left(q^{-1}\right)=\sum_{y \in[u, v]^{\backslash J}} Z_{u, y}^{J, q}(q) P_{y, v}(q) .
$$


Proof From Corollary 4.3 and Theorem 2.10 we find

$$
\begin{aligned}
\sum_{y \in[u, v]^{\backslash J}} Z_{u, y}^{J, q} P_{y, v} & =\sum_{y \in[u, v]^{\backslash J}} R_{u, y} P_{y, v}-\sum_{y \in[u, v]^{\backslash J}}(-1)^{\ell\left(y_{J}\right)} R_{u, y^{J}} P_{y, v} \\
& =\sum_{y \in[u, v]^{J J}} R_{u, y} P_{y, v}-\sum_{w \in W^{J}} R_{u, w} \sum_{y \in W_{J} \backslash\{e\}}(-1)^{\ell(y)} P_{y w, v} \\
& =\sum_{y \in[u, v]^{\backslash J}} R_{u, y} P_{y, v}+\sum_{w \in[u, v] \cap W^{J}} R_{u, w} P_{w, v} \\
& =q^{\ell(u, v)} P_{u, v}\left(q^{-1}\right),
\end{aligned}
$$

because, by Proposition 6.10, $\sum_{y \in W_{J} \backslash\{e\}}(-1)^{\ell(y)} P_{y w, v}=-P_{w, v}$.

Remark 7.2 Note that the KLS-function of $W \backslash W^{J}$ relative to $Z^{J, q}$ does not depend on $J$.

Remark 7.3 If $u, v \in W \backslash W^{J}$ and $u<v^{J}$ then $[u, v]^{\backslash J} \subset[u, v]$, by Lemma 5.1. Therefore, in this case, Theorem 7.1 gives a recursion for the computation of the Kazhdan-Lusztig polynomials $P_{u, v}$ alternative to the one of Theorem 2.10.

Remark 7.4 Theorem 7.1 gives another way to deduce the Möbius function of the poset $W \backslash W^{J}$. In fact, if $v, w \in W \backslash W^{J}$, from Theorem 7.1 and Theorem 2.10 we have that $\sum_{y \in[v, w]^{\backslash J}} Z_{v, y}^{J, q}(0)=0$; so $Z_{v, w}^{J, q}(0)=\mu^{\backslash J}(v, w)$, and the result follows from Corollary 4.11.

We consider now the case $x=-1$; we define $Z^{J,-1} \in \tilde{I}\left(W \backslash W^{J}\right)$ by $Z^{J,-1}(u, v):=Z_{u, v}^{J,-1}$ for all $u, v \in W \backslash W^{J}$.

Lemma 7.5 The element $f \in \tilde{I}\left(W \backslash W^{J}\right)$, which is defined by $f(u, v):=$ $(-q)^{\ell(u, v)} P_{u, v}\left(q^{-1}\right)$ for all $u, v \in W \backslash W^{J}$, is $Z^{J,-1}$-totally acceptable, i.e.

$$
(-1)^{\ell(u, v)} P_{u, v}(q)=\sum_{y \in[u, v]^{J}} Z_{u, y}^{J,-1}(q)(-q)^{\ell(y, v)} P_{y, v}\left(q^{-1}\right),
$$

for all $u, v \in W \backslash W^{J}$.

Proof By Proposition 4.12 and Theorem 7.1 we have

$$
\begin{aligned}
\sum_{y \in[u, v]^{\backslash J}} & Z_{u, y}^{J,-1}(q)(-q)^{\ell(y, v)} P_{y, v}\left(q^{-1}\right)= \\
= & \sum_{y \in[u, v]^{\backslash J}}(-q)^{\ell(u, y)} Z_{u, y}^{J, q}\left(q^{-1}\right)(-q)^{\ell(y, v)} P_{y, v}\left(q^{-1}\right) \\
= & (-q)^{\ell(u, v)} \sum_{y \in[u, v]^{\backslash J}} Z_{u, y}^{J, q}\left(q^{-1}\right) P_{y, v}\left(q^{-1}\right)=(-q)^{\ell(u, v)} q^{-\ell(u, v)} P_{u, v}(q) .
\end{aligned}
$$


Corollary 7.6 The element $Z^{J,-1} \in \tilde{I}\left(W \backslash W^{J}\right)$ is a $\left(W \backslash W^{J}\right)$-kernel, and there exists a unique $Z^{J,-1}$-totally acceptable element $\tilde{P}^{J,-1} \in I_{1 / 2}\left(W \backslash W^{J}\right)$.

Proof Since $(-q)^{\ell(u, v)} P_{u, u}\left(q^{-1}\right)=1$ for all $u \in W \backslash W^{J}$, this is an invertible element of $\tilde{I}\left(W \backslash W^{J}\right)$, and it is $Z^{J,-1}$-totally acceptable, by Lemma 7.5. The last assertion follows from Theorem 2.12.

We call $\tilde{P}^{J,-1}$ the KLS-function of $W \backslash W^{J}$ relative to the kernel $Z^{J,-1}$.

Remark 7.7 Although the KLS-function of $W \backslash W^{J}$ relative to the kernel $Z^{J, q}$ is known, being equal to $P_{v, w}$ by Theorem 7.1, we do not know an expression for $\tilde{P}_{v, w}^{J,-1}$ in terms of known polynomials.

The next example shows that the polynomials $\tilde{P}^{J,-1}$ can have negative coefficients.

Example 7.8 As in Example 4.5, take $v=324156$ and $w=546132$ in $W \backslash W^{S \backslash\left\{s_{3}\right\}}$. Then

$$
\tilde{P}_{v, w}^{S \backslash\left\{s_{3}\right\},-1}=-5 q^{2}+2 q .
$$

For each $x \in\{-1, q\}$ we define an element $\mathscr{Z}^{J, x} \in \tilde{I}\left(W \backslash W^{J}\right)$ by letting

$$
\mathscr{Z}^{J, x}(u, v):=(-1)^{\ell(u, v)} Z_{u, v}^{J, x},
$$

for all $u, v \in W \backslash W^{J}, u \leqslant v$. Then, from Theorem 2.13 and Corollary 4.12, we find

Corollary 7.9 Let $(W, S)$ be a Coxeter system, $J \subseteq S, x \in\{-1, q\}$, and $u, v \in$ $W \backslash W^{J}, u \leqslant v$. Then

$$
\tilde{P}_{u, v}^{J, x}=\sum_{\mathscr{C} \in \mathbf{M}^{\backslash J}(u, v)}\left(\mathscr{Z}^{J, q-1-x}\right)_{\mathscr{C}}
$$

where $\mathbf{M}^{\backslash J}(u, v)$ denotes the set of all multichains in $W \backslash W^{J}$ from $u$ to $v$.

Acknowledgments I would like to thank my advisor Prof. Francesco Brenti for his attentive guidance during this work, which is part of my $\mathrm{PhD}$ thesis.

\section{References}

1. Björner, A.: Posets, regular CW complexes and Bruhat order. Eur. J. Combin. 5(1), 7-16 (1984)

2. Björner, A., Brenti, F.: Combinatorics of Coxeter Groups. Graduate Texts in Mathematics, vol. 231. Springer, Berlin Heidelberg (2005)

3. Björner, A., Wachs, M.: Bruhat order of Coxeter groups and shellability. Adv. Math. 43(1), 87-100 (1982)

4. Brenti, F.: Twisted incidence algebras and Kazhdan-Lusztig-Stanley functions. Adv. Math. 148, 44-74 (1999)

5. Deodhar, V.V.: Some characterizations of Bruhat ordering on a Coxeter group and determination of the relative Möbius function. Invent. Math. 39(2), 187-198 (1977) 
6. Deodhar, V.V.: On some geometric aspects of Bruhat orderings. The parabolic analogue of KazhdanLusztig polynomials. J. Algebra 111(2), 483-506 (1987)

7. Deodhar, V.V.: Duality in parabolic set up for questions in Kazhdan-Lusztig theory. J. Algebra 142, 201-209 (1991)

8. Humphreys, J.E.: Reflection Groups and Coxeter Groups. Cambridge Studies in Advanced Mathematics, vol. 29. Cambridge University Press, Cambridge (1990)

9. Kazhdan, D., Lusztig, G.: Representations of Coxeter groups and Hecke algebras. Invent. Math. 53(2), 165-184 (1979)

10. Lusztig, G.: Characters of reductive groups over a finite field. Annals of Mathematics Studies, vol. 107. Princeton University Press, Princeton (1984)

11. Pesiri, A.: Combinatorial properties of Temperley-Lieb algebras. $\mathrm{PhD}$ thesis, Università degli Studi di Roma "Tor Vergata" (2011)

12. Pesiri, A.: Combinatorial properties of the Temperley-Lieb algebra of a Coxeter group. J. Algebraic Combin. 37, 717-736 (2013)

13. Stanley, R.P.: Subdivisions and local h-vectors. J. Amer. Math. Soc. 5, 805-851 (1992)

14. Stanley, R.P.: Enumerative Combinatorics, vol. 1. Cambridge University Press, Cambridge (1997) 